\title{
Designing for Gender-Role Differences Through the Lens of Self-Determination Theory
}

\author{
Gabriela Villalobos-Zúñiga \\ gabriela.villalobos@unil.ch \\ University of Lausanne \\ Lausanne, Switzerland
}

\begin{abstract}
Persuasive apps aim to contribute to the behavior change process of individuals. Some of these apps include personalization strategies that contribute to more effective changes in their behavior. Genderrole differences are an aspect that can be further explored to improve app tailoring. The Self-Determination Theory serves as a theoretical guide to design personalized persuasive apps that support genderrole differences. This position paper sparkles the discussion around design ideas that might support these gender-role differences.
\end{abstract}

\section{CCS CONCEPTS}

- Human-centered computing $\rightarrow$ HCI design and evaluation methods.

\section{KEYWORDS}

gender-inclusive software; gender biases; personalization; behaviorchange; persuasive technology

\section{ACM Reference Format:}

Gabriela Villalobos-Zúñiga. 2021. Designing for Gender-Role Differences Through the Lens of Self-Determination Theory. In Adjunct Proceedings of the 29th ACM Conference on User Modeling, Adaptation and Personalization (UMAP '21 Adjunct), fune 21-25, 2021, Utrecht, Netherlands. ACM, New York, NY, USA, 4 pages. https://doi.org/10.1145/3450614.3464622

\section{INTRODUCTION}

Persuasive apps (mobile applications created with the intention to serve as tools for positive behavior change in individuals), have a high presence in the main app markets. Example of these apps include: MyFitnessPal [19] and Endonomdo [3]. Many of these persuasive apps adopt strategies of customization of their app functions (e.g., rewards and comparison). Taking into account the specific characteristics of their target users contributes to a better acceptance of the app from the users and to more effective behavior change results on the users [7, 13]. Many of these personalizations occur in the spaces of personality $[6,10]$, culture [12], and social factors [15]. Furthermore, exploring the space of gender-role differences has potential to bring improvement to the current persuasive technologies by creating technology that considers these

Permission to make digital or hard copies of all or part of this work for personal or classroom use is granted without fee provided that copies are not made or distributed for profit or commercial advantage and that copies bear this notice and the full citation on the first page. Copyrights for components of this work owned by others than the author(s) must be honored. Abstracting with credit is permitted. To copy otherwise, or republish, to post on servers or to redistribute to lists, requires prior specific permission and/or a fee. Request permissions from permissions@acm.org.

UMAP '21 Adjunct, fune 21-25, 2021, Utrecht, Netherlands

(c) 2021 Copyright held by the owner/author(s). Publication rights licensed to ACM ACM ISBN 978-1-4503-8367-7/21/06 . \$ $\$ 15.00$

https://doi.org/10.1145/3450614.3464622 differences. Therefore in this position paper I propose to study and understand how to leverage on these gender-role differences to create designs that consider this variations and consequently produce inclusive and effective persuasive technology. A theoretical framework that can support this technology design is the SelfDetermination Theory (SDT) [2]. In the following sections, I introduce the concepts of technology personalization and gender-role differences and provide illustrative examples of the suggested design.

\section{TECHNOLOGY PERSONALIZATION}

Human beings are naturally diverse: Individuals belong to different cultures, develop in different societies, and have diverse personalities. This diversity should be considered when designing technological solutions, especially when these are intended to modify the individual's behavior. Research has shown that the "one-size-fitsall" approach is less effective than personalizing persuasive technologies $[7,13]$. This is because different types of individuals find motivation due to different strategies [14]. Therefore, it is imperative to deeply study this human diversity to have solid information to create personalized strategies. Some commonly used persuasive strategies include: customization, simulation, self-monitoring and feedback, suggestion, personalization, simulation, praise, reward, comparison, competition, and cooperation $[4,11]$. An area where this offering of tailored content can be explored is gender-role differences.

\section{GENDER-ROLE DIFFERENCES}

Gender-role differences have been studied in various domains, for instance Lauderdale et al. [9], researched on how gender differences affected motivation towards physical activity among college students. They found significant gender differences with males responding more positively to intrinsic motivation. Oyibo et al. [17] studied persuasive features that foster adoption of fitness apps. They found that for males, rewards have a positive relationship with the intention to use an app; while for females cooperation and competition are the features that produce this positive relationship. In another study, Oyibo et al. [16] analyzed gender preferences and differences in behavior modeling in fitness applications; finding differences in exercise-type preferences, perceived self-efficacy and projected exercise performance level. In the job domain, particularly in the health sector, Gómez-Baya et al. [5] noted gender differences with respect to the levels of psychological well-being, health problems, and basic psychological needs satisfactions. In another study focused on technology, Klenk and colleagues [8], noticed significant differences between female and male participants where females found the gratification of achieving goals and enjoyment more 
important for their motivation than males. They also noticed that males appreciate more sharing their activity results than females.

\section{SELF-DETERMINATION THEORY}

The Self-Determination Theory (SDT) is a theory of human motivation that can guide understanding and tailoring persuasive technology for gender-role differences. SDT states that people have different levels of motivation that go from extrinsic to intrinsic motivation [2]. Additionally, the theory describes three Basic Psychological Needs (or BPN), that when satisfied by the context in which the individual develops, leads to a self-determined action. The BPNs are defined as follows:

(1) Autonomy "refers to feeling willingness and volition with respect to one's behavior. The need for autonomy describes the need of individuals to experience self-endorsement and ownership of their actions." [18, p. 86]

(2) Competence "refers to feeling effective in one's interactions with the social environment-that is, experiencing opportunities and support for the exercise, expansion, and expression of an individual's capacities and talents." [18, p. 86]

(3) Relatedness "refers to both experiencing others as responsive and sensitive and being able to be responsive and sensitive to them-that is, feeling connected and involved with others and having a sense of belonging." [18, p. 86]

\section{INCLUSIVE PERSUASIVE DESIGN}

Inclusion is "the act of allowing many different types of people to do something and treating them fairly and equally" [1]. In persuasive technology, designing for gender inclusiveness is of great significance because achieving changes in behavior with technology that does not adapt to their users' preferences results useless. To approach to this gender personalization, Oyibo and colleagues [16] conducted a study on exercise type preferences. They concluded that $80 \%$ of their study females population preferred a small group of exercises. Oyibo et al. suggest to designers creating female fitness apps to prioritize these exercises over the ones that were not categorized with high priority by the female participants. Incorporating this suggestion into the software design will satisfy the fitness preference of a majority but, unfortunately, will ignore the fitness preference of a minority of female users.

Another consideration for gender inclusiveness is that during data collection processes, participants have the option to select the non-binary choice whenever they do not feel identified with male or female. This practice will yield more representative samples, which can be used to create more robust inclusive designs.

In this position paper, I argue that to produce effective persuasive technology is not only necessary to design for gender inclusion, but also to consider psychological theories of human motivation. The Self-Determination Theory fits well in persuasive design because of its focus on self-determined action.

\subsection{Designing to Support the SDT Basic Psychological Needs}

In the following paragraphs, I will provide illustrative examples of incorporating SDT into the app design process to yield inclusive, effective persuasive designs.
Autonomy: Consider the design of a female inclusive fitness app. Seeing the previously mentioned findings from Oyibo's and colleagues' work [16], we know that $80 \%$ of the female's study population prefers a particular type of exercise. Therefore, we proceed to design a goal setting feature, which we know from previous literature supports the need of autonomy [20] (See Figure 1a). This feature includes a list of exercises to choose and considers $80 \%$ of the female population, however, what about the other $20 \%$ ? Because we do not have data about the exercise preferences of this minority (See Figure 1b), I suggest two paths: 1: Follow a data collection process which provides data about the exercise type tastes and preferences of the minority of females. 2: Include the full set of exercise apps and allow the individual to choose the ones that match their preferences (See Figure 1c).
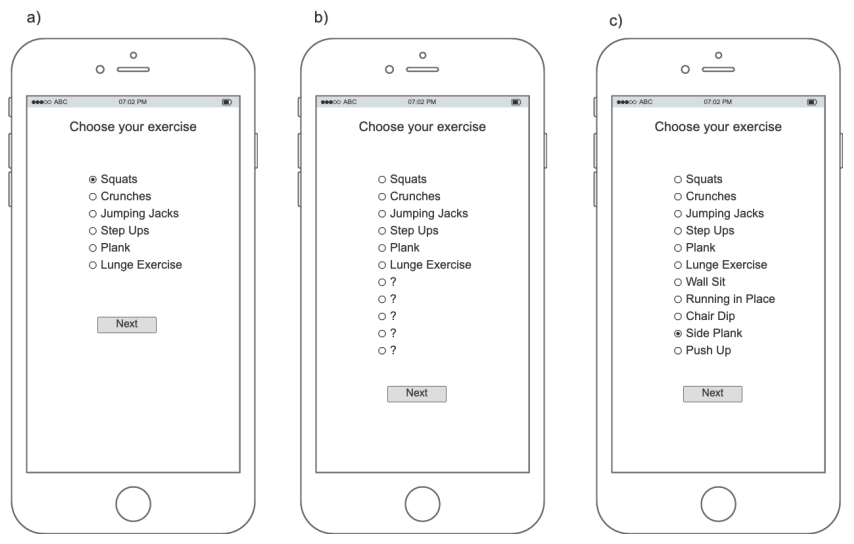

Figure 1: (a) Goal setting feature with exercise options for $80 \%$ of female population. (b) Goal setting feature representing missing features for $20 \%$ of female population. (c) Goal setting feature with an inclusive design.

Competence: Consider the design of male and female inclusive fitness app. Taking the findings from Oyibo et al. [17] about persuasive features with a positive intention to use, we know that rewards contribute to males using an app but not females. From this same research findings we know that females prefer self-monitoring, therefore we include this feature as a competence support [20]. As a result we have an inclusive design that includes rewards and goal setting (See Figure 2).

Relatedness: Consider the design of a male and female inclusive fitness app. We know from Klenk et al. [8] results that $89.4 \%$ of the population of males in their study used the sharing results feature on Runtastic. With this information we might include the performance-sharing[20] feature as a relatedness supportive feature. However, we will end up in a similar situation to the autonomy example mentioned above: we ignore the preferences of about $11 \%$ of the male population. Therefore to reach full support, I suggest following the same approach as in the autonomy example. Next, to support relatedness for females, the designer might want to consider the leaderboard feature as defined in previous literature [20] (See Figure 3). 


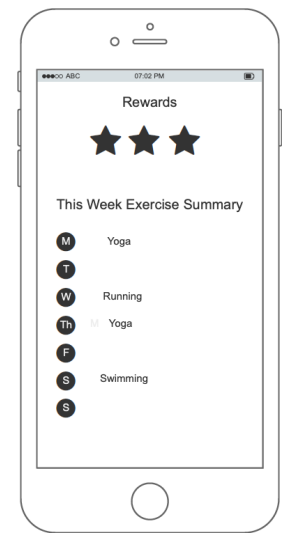

Figure 2: Rewards on the top represented by stars and SelfMonitoring on the bottom represented by a list of exercises performed during the week.

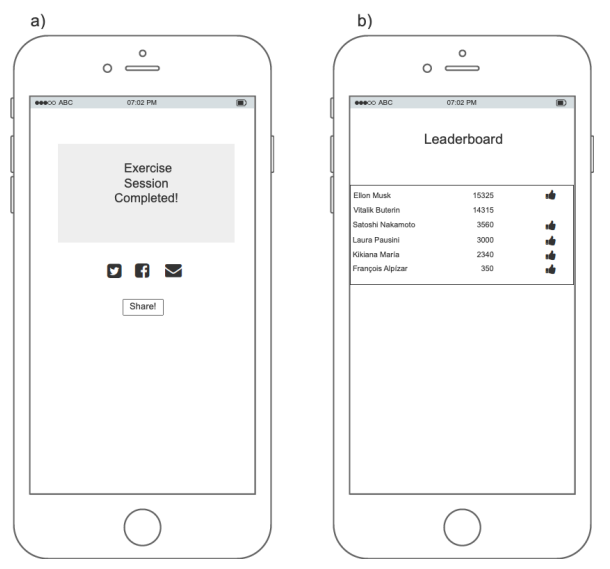

Figure 3: (a) Performance-sharing displaying options to share on social media. (b) Leaderboard showing a list of users in ranked order.

In summary, the goal of the proposed design strategy is two-fold: (1) Design trying to eliminate barriers and enhancing features so that all genders (including their diversity) can make profound use of technology [21]. This is, avoiding the design by segmentation, creating one software version for males and another for females. (2) By supporting the basic psychological needs through features that are well received by each gender, we will have persuasive apps which are more effective because individuals are driven by selfdetermined behavior. These two approaches have the potential to produce inclusive, effective persuasive technology.

\section{CONTRIBUTION AND GOALS IN PARTICIPATING IN THE WORKSHOP}

In this position paper, I want to discuss the importance of tailoring persuasive interventions for gender-role differences, as mentioned earlier. Given that these differences exist, I argue that persuasive apps can benefit from higher effectiveness if these are studied, understood, and included. Consequently, researchers learn how to incorporate this diversity into technological solutions. For this workshop's purpose, I want to brainstorm on design ideas in which technology can be more inclusive to this gender-role differences using as a framework the Self-Determination Theory.

\section{REFERENCES}

[1] 2021. Cambridge Dictionary. Cambridge University Press. https://dictionary. cambridge.org/dictionary/english/inclusion

[2] Edward L Deci and Richard M Ryan. 1985. Intrinsic motivation and selfdetermination in human behavior. Springer Science \& Business Media, New York, US.

[3] Endomondo. 2020. The personal trainer in your pocket. (2020). https://www. endomondo.com/

[4] BJ Fogg. 2003. Persuasive Technology: Using Computers to Change What We Think and Do. Morgan Kaufmann Publishers. San Francisco (2003).

[5] Diego Gómez-Baya, Ana M. Lucia-Casademunt, and José A. Salinas-Pérez. 2018. Gender differences in psychological well-being and health problems among european health professionals: Analysis of psychological basic needs and job satisfaction. International fournal of Environmental Research and Public Health 15, 7 (2018). DOI : http://dx.doi.org/10.3390/ijerph15071474

[6] Sajanee Halko and Julie A Kientz. 2010. Personality and persuasive technology: an exploratory study on health-promoting mobile applications. In International conference on persuasive technology. Springer, 150-161.

[7] Maurits Kaptein, Panos Markopoulos, Boris De Ruyter, and Emile Aarts. 2015. Personalizing persuasive technologies: Explicit and implicit personalization using persuasion profiles. International Journal of Human-Computer Studies 77 (2015), 38-51.

[8] Saskia Klenk, Doreen Reifegerste, and Rebecca Renatus. 2017. Gender differences in gratifications from fitness app use and implications for health interventions. Mobile Media and Communication 5, 2 (2017), 178-193. DOI : http://dx.doi.org/10. $1177 / 2050157917691557$

[9] Michael E. Lauderdale, Sami Yli-Piipari, Carol C. Irwin, and Todd E. Layne. 2015. Gender Differences Regarding Motivation for Physical Activity Among College Students: A Self-Determination Approach. The Physical Educator 72 (2015), 153-172. DOI : http://dx.doi.org/10.18666/tpe-2015-v72-i5-4682

[10] Oded Nov, Ofer Arazy, Claudia López, and Peter Brusilovsky. 2013. Exploring personality-targeted UI design in online social participation systems. In Proceedings of the SIGCHI Conference on Human Factors in Computing Systems. 361-370.

[11] Harri Oinas-Kukkonen and Marja Harjumaa. 2008. A systematic framework for designing and evaluating persuasive systems. In International conference on persuasive technology. Springer, 164-176.

[12] Rita Orji. 2016. The impact of cultural differences on the persuasiveness of influence strategies. In Proceedings of the 11th International Conference on Persuasive Technology. 38-41.

[13] Rita Orji, Regan L Mandryk, and Julita Vassileva. 2017. Improving the efficacy of games for change using personalization models. ACM Transactions on ComputerHuman Interaction (TOCHI) 24, 5 (2017), 1-22.

[14] Rita Orji, Regan L Mandryk, Julita Vassileva, and Kathrin M Gerling. 2013. Tailoring persuasive health games to gamer type. In Proceedings of the sigchi conference on human factors in computing systems. 2467-2476.

[15] Kiemute Oyibo and Julita Vassileva. 2017. Investigation of social predictors of competitive behavior in persuasive technology. In International Conference on Persuasive Technology. Springer, 279-291.

[16] Kiemute Oyibo and Julita Vassileva. 2020a. Gender preference and difference in behavior modeling in fitness applications: A mixed-method approach. Multimodal Technologies and Interaction 4, 2 (2020), 1-31. DOI : http://dx.doi.org/10.3390/ miti4020021

[17] Kiemute Oyibo and Julita Vassileva. 2020b. Persuasive features that drive the adoption of a fitness application and the moderating effect of age and gender. Multimodal Technologies and Interaction 4, 2 (2020), 1-19. DOI : http://dx.doi.org/ $10.3390 / \mathrm{mti} 4020017$

[18] Richard M. Ryan and Edward L. Deci. 2017. Self-Determination Theory Basic Psychological Needs in Motivation, Development and Wellness. THE GUILFORD PRESS, 370 Seventh Avenue, Suite 1200, New York, NY 10001. 770 pages. https://www. guilford.com/books/Self-Determination-Theory/Ryan-Deci/9781462528769

[19] Under Armour Inc. 2020. MyFitnessPal: Fitness starts with what you eat. (2020). https://www.myfitnesspal.com/

[20] Gabriela Villalobos-Zúñiga and Mauro Cherubini. 2020. Apps That Motivate: a Taxonomy of App Features Based on Self-Determination Theory. International Journal of Human-Computer Studies 140, 102449 (2020), 102449. DOI : http://dx. doi.org/https://doi.org/10.1016/j.ijhcs.2020.102449 
[21] Mihaela Vorvoreanu, Lingyi Zhang, Yun Han Huang, Claudia Hilderbrand, Zoe Steine-Hanson, and Margaret Burnett. 2019. From gender biases to genderinclusive design: An empirical investigation. Conference on Human Factors in
Computing Systems - Proceedings (2019), 1-14. DOI : http://dx.doi.org/10.1145/ 3290605.3300283 